\title{
O trabalho na central de material: repercussões para a saúde dos trabalhadores de enfermagem
}

\author{
Working at central supply and sterilization: health implications for nursing workers \\ El trabajo en la central de material: implicaciones para la salud de los trabajadores de enfermería
}

\author{
Carolina Cabral Pereira da CostaI; Norma Valéria Dantas de Oliveira Souza ; Patrícia Alves dos Santos Silvalil; \\ Elias Barbosa de Oliveira ${ }^{\text {IV }}$ Manoel Luís Cardoso Vieira ${ }^{V}$
}

\begin{abstract}
RESUMO: Objetivou-se, neste trabalho, analisar a configuração da organização e do processo de trabalho na Central de Material e Esterilização, na perspectiva de interferência no processo saúde-doença dos trabalhadores de enfermagem. Trata-se de uma pesquisa qualitativa, descritiva, realizada em 2013, com 34 trabalhadores de enfermagem de uma central de material de hospital universitário do Rio de Janeiro. Utilizou-se a entrevista semiestruturada, associada à observação não participante. As informações coletadas foram transcritas, analisadas e interpretadas à luz da análise temática de conteúdo. Os entrevistados referem que, durante as atividades laborais, estão expostos a riscos e agravos relacionados a fatores ergonômicos, biológicos, entre outros, e apontam que a repetitividade das tarefas pode acarretar prejuízos à saúde dos trabalhadores. Conclui-se que é relevante a sensibilização dos gestores e trabalhadores para esse contexto de trabalho, que conduz às manifestações indesejáveis na saúde e ao desenvolvimento de doenças nos trabalhadores.
\end{abstract}

Palavras-Chave: Enfermagem; saúde do trabalhador; enfermagem do trabalho; esterilização.

\begin{abstract}
This qualitative, descriptive study examined the hospital Central Supply and Sterilization (CSS) organization and work process from the perspective of its role in health-disease processes among nursing workers. In 2013, 34 nursing workers at a university hospital CSS in Rio de Janeiro underwent semi-structured interviews, associated with non-participant observation. The data were transcribed, analyzed and interpreted in the light of thematic content analysis. Respondents reported that, during work activities, they were exposed to risks and injuries related to ergonomic, biological, and other factors, and pointed out that the repetitive tasks can lead to health problems for workers. It was concluded that it is important to raise managers' and workers' awareness to this work environment, which is conducive to undesirable health conditions and to the development of diseases in workers. Keywords: Nursing; workers' health; nursing work; sterilization.
\end{abstract}

RESUMEN: El objetivo de este estudio fue analizar la configuración de la organización y del proceso de trabajo en la Central de Material y Esterilización, dentro de la perspectiva de interferencia en el proceso salud-enfermedad de los trabajadores de enfermería. Se trata de un estudio cualitativo, descriptivo, realizado en 2013, junto a 34 trabajadores de enfermería de una central de material de un hospital universitario de Río de Janeiro. Se utilizó una entrevista semiestructurada, asociada con la observación no participante. Las informaciones recolectadas fueron transcritas, analizadas e interpretadas a la luz del análisis de contenido temático. Los encuestados informan que, durante las actividades de trabajo, están expuestos a riesgos y agravios relacionados con los factores biológicos, ergonómicos, entre otros, y señalan que la repetición de tareas puede conducir a perjuicios a la salud de los trabajadores. Se concluye que es importante la sensibilización de los gestores y los trabajadores para este contexto de trabajo que conduce a manifestaciones indeseables en la salud y al desarrollo de enfermedades en los trabajadores.

Palabras Clave: Enfermería; salud del trabajador; enfermería del trabajo; esterilización.

\section{INTRODUÇÃO}

As centrais de material e esterilização (CME) têm a missão de servir de apoio às unidades assistenciais, pois esterilizam e desinfetam materiais hospitalares permanentes e preparam materiais de consumo, fornecendo-os ao centro cirúrgico e aos demais setores das unidades hospitalares ${ }^{1}$. A maioria das CME é caracterizada por sistemas de ventilação incipientes, espaços físicos reduzidos e isolados, mobiliário ergonomicamente inapropriado, entre outros aspectos que confluem para a deterioração da saúde dos trabalhadores² .

${ }^{\mathrm{I}}$ Doutoranda em Enfermagem pela Universidade do Estado do Rio de Janeiro. Enfermeira do Instituto de Cardiologia Aloysio de Castro. Professora do Centro Universitário Celso Lisboa. Rio de Janeiro, Brasil. E-mail: carolcuerj@hotmail.com.

IIDoutora em Enfermagem. Professora Associada e Permanente da Pós-Graduação stricto sensu em Enfermagem da Universidade do Estado do Rio de Janeiro. Brasil. E-mail: norval_souza@yahoo.com.br.

IIIMestranda em Enfermagem pela Universidade do Estado do Rio de Janeiro. Brasil. E-mail: papatyenf@gmail.com.

Iv Pós-Doutor em Álcool e Drogas. Professor Adjunto e Permanente da Pós-Graduação stricto-sensu em Enfermagem da Universidade do Estado do Rio de Janeiro. Brasil.E-mail: eliasbo@gmail.com.

${ }^{\mathrm{v}}$ Mestre em Enfermagem. Especialista em Enfermagem do Trabalho e em Centro Cirúrgico e Central de Material e Esterilização. Rio de Janeiro, Brasil. E-mail: mlcv22@bol.com.br. 
A partir de uma busca nas bases de dados da Biblioteca Virtual em Saúde, evidencia-se uma incipiente produção científica envolvendo o tema, fortalecendo, assim, a justificativa do estudo. No entanto, a primeira e mais relevante justificativa ancora-se no fato de o estudo buscar desvendar os meandros de um espaço de trabalho da enfermagem que vem sendo esquecido pelos gestores, em termos tanto de pesquisa quanto da necessidade da melhoria das condições de trabalho.

Assim, este estudo fortalece a profissão, incentivando o ensino, a assistência e pesquisas relacionados à saúde do trabalhador, vislumbrando-se estratégias mais efetivas para a dinâmica de trabalho nesse cenário, a fim de assegurar o bem-estar do coletivo profissional. Além disto, contribui para ampliar a compreensão sobre alguns dos fatores que têm causado o adoecimento físico e/ou mental dos trabalhadores de enfermagem, somado à possibilidade de uma compreensão mais aprofundada sobre os efeitos do trabalho na CME no corpo do trabalhador.

Logo, este estudo tem por objeto as repercussões do trabalho na central de material para a saúde dos trabalhadores de enfermagem e objetivou-se analisar a configuração da organização e do processo de trabalho na CME, na perspectiva de interferência no processo saúde-doença dos trabalhadores de enfermagem.

\section{REVISÃo DE LITERATURA}

O trabalho pode ser entendido como produtor de valores de uso, expressão de uma relação metabólica entre o ser social e a natureza. Ele deveria ser visto como fonte de prazer e realização, pois favorece a socialização e a busca pela autorrealização, além de garantir a subsistência. Entretanto, o trabalho não representa sempre a satisfação pessoal e a valorização do ser humano, embora se destaque como forma de satisfazer as necessidades materiais básicas ${ }^{3}$.

Atualmente, com a vigência do modelo neoliberal, verificam-se repercussões negativas para os trabalhadores, como o medo do desemprego, com redução dos postos de trabalho; multiplicidade de vínculos laborais, com predominância de formas de contratos precários; redução e/ou fim de direitos trabalhistas. Tais circunstâncias fazem com que os trabalhadores se submetam a condições laborais adversas e passem a ficar limitados a realizarem reivindicações, o que enfraquece, portanto, as lutas coletivas por mudanças. A precarização do trabalho é um fenômeno que acomete a maioria dos trabalhadores pela desregulamentação e pela perda dos direitos laborais e sociais, o que, certamente, causa sofrimento e aumenta a vulnerabilidade às doenças ocupacionais ${ }^{4}$.

Esse contexto de adversidades impõe aos gestores da assistência hospitalar o desafio de estabelecer políticas que incidam sobre a organização do trabalho as quais não se limitem simplesmente ao controle dos riscos já conhecidos e identificados - como os biológicos, físicos, químicos -, mas que protejam, também, a dimensão subjetiva dos trabalhadores frente ao sofrimento psíquico imposto por tal modelo produtivo, com o desafio de assegurar a produtividade com excelência ${ }^{5}$.

A CME é uma unidade essencial no cenário hospitalar, sendo responsável pela recepção, expurgo, limpeza, descontaminação, preparo, esterilização, guarda e distribuição dos materiais utilizados por diversos setores na estrutura organizacional ${ }^{6}$. Entretanto, o processo de trabalho nas CME acarreta sofrimento psíquico aos trabalhadores, expressos por sentimentos de abandono, tristeza, doenças psicossomáticas e mentais. Acrescenta-se, ainda, o presenteísmo no trabalho e o descontentamento diante da falta de reconhecimento e de valorização das atividades desses trabalhadores no contexto hospitalar ${ }^{7,8}$.

O trabalho executado na CME é repetitivo e requer atenção daqueles que o realizam; assim, há dificuldade em manter no setor profissionais estimulados e em sincronia com as tarefas a serem realizadas. Além disto, na maioria das CME, existem riscos ocupacionais que podem ocasionar doenças e incapacidades temporárias ou permanentes?.

\section{Metodologia}

Esta é uma pesquisa qualitativa e descritiva. $\mathrm{O}$ cenário é uma CME de um hospital universitário, no Rio de Janeiro. Os participantes do estudo são 34 profissionais de enfermagem (nove enfermeiras e 25 técnicos de enfermagem) que trabalhavam na referida CME.

Os critérios de inclusão utilizados são: tempo de atuação no cenário igual ou superior a seis meses; e tipo de vínculo com o hospital, podendo o profissional ser contratado formalmente pela Consolidação da Legislação Trabalhista (CLT) ou, ainda, trabalhar sob outra forma de contratação (contrato precário), em quaisquer escalas de plantão.

Já os critérios de exclusão são: estar de férias ou de licença de qualquer natureza durante o período de coleta de dados; e ser residente de enfermagem. Este critério de exclusão justifica-se porque, apesar de a instituição possuir um Programa de Residência em Enfermagem, seus residentes passam apenas um curto período na unidade, podendo, então, comprometer a percepção da organização e do processo laboral devido a uma visão superficial desse trabalho.

Para desenvolver a proposta de investigação, utilizou-se a entrevista individual do tipo semiestruturada. Além disso, usou-se a observação não participante, norteada por um formulário com aspectos referentes à estrutura física da CME e ao processo e à organização laboral, os quais podem interferir no processo saúde- 
doença dos trabalhadores de enfermagem. Totalizam-se 20 horas de registro de campo, realizadas em dias e horários distintos.

A coleta de dados foi efetuada entre março e maio de 2013; o tempo médio de entrevistas abrangeu 15 minutos, sendo todas realizadas por apenas uma pesquisadora. A fim de atender às exigências éticas, o projeto do estudo foi enviado ao Comitê de Ética em Pesquisa (CEP) da Universidade do Estado do Rio de Janeiro, sendo aprovado no referido CEP sob protocolo $\mathrm{n}^{\mathrm{O}}$ 081.3.2012.

Ainda, cada participante recebeu e assinou um Termo de Consentimento Livre e Esclarecido, e preservou-se a privacidade dos participantes, identificando-os por um código alfanumérico nas descrições dos relatos (E para enfermeiros e TE para técnicos de enfermagem; a numeração seguiu a ordem cronológica das transcrições das entrevistas). Todos esses procedimentos estão em consonância com a Resolução n ${ }^{\circ}$ 466/2012 do Conselho Nacional de Saúde/Ministério da Saúde ${ }^{10}$.

As informações coletadas foram transcritas, analisadas e interpretadas à luz da análise temática de conteúdo ${ }^{11}$. Na categorização dos dados, seguiram-se os passos: pré-análise (leitura flutuante); constituição do corpus; formulação de hipóteses e objetivos; exploração do material; e tratamento dos resultados obtidos $^{11}$. Os dados coletados por meio da observação foram inseridos na análise e discussão, com o objetivo de reafirmar, enriquecer ou, até mesmo, evidenciar a relação entre os discursos e a observação realizada. Assim, organizam-se duas categorias para este estudo, a saber: processo saúde-doença dos trabalhadores: fatores determinantes e condicionantes; e fatores que resgatam e asseguram a saúde do trabalhador.

\section{Resultados e Discussão}

\section{Processo saúde-doença dos trabalhadores: fatores determinantes e condicionantes}

Os resultados tratam de situações pertinentes às características do processo e da organização do trabalho, as quais predispõem os trabalhadores de enfermagem à saúde ou à doença.

Nesse contexto, os participantes relatam que compreendem a exposição a riscos e agravos relacionados a fatores ergonômicos no cotidiano de trabalho.

O nosso serviço fica em dois andares, o material é pesado, você tira aquele material e tem que subir uma rampa para levar esse material até o outro andar e ser esterilizado. A gente pega muito peso, faz mal para coluna. (TE6)

Eu acho que o peso excessivo que a gente carrega prejudica a saúde, essas caixas superpesadas de material consignado da ortopedia, por exemplo. Isso influi muito e prejudica a coluna de qualquer ser humano. (TE1)
Durante as observações de campo, constatou-se que os profissionais de enfermagem são submetidos à sobrecarga de peso, uma vez que o material de trabalho na CME é pesado e os subsetores são distantes uns dos outros. Vale ressaltar que os profissionais precisam subir e descer escadas, pois não há rampas e/ou elevadores; também não há equipamentos de transporte que minimizem a sobrecarga de peso.

É preciso destacar que a carga física é muito elevada, já que a manipulação de peso é constante, pois há necessidade de carregar as caixas de instrumentais cirúrgicos, o que demanda força física intensa. Tais atividades, se realizadas com posturas inadequadas, podem potencializar ainda mais a ocorrência de sérios problemas de coluna e/ou lesões osteomusculares ${ }^{12}$.

Merece destaque a repetição das ações realizadas no decorrer do trabalho, como um fator que pode causar algum tipo de adoecimento.

Aqui na CME, o trabalho é bastante repetitivo, exaustivo, pode levar a alguma lesão. A gente faz sempre as mesmas coisas, às vezes cansa. Dá até vontade de sair daqui. (E1)

O processo laboral na CME tem particularidades decorrentes de sua organização e desenvolvimento, expondo os trabalhadores a cargas de trabalho específicas, que trazem repercussões negativas à saúde física e mental dos trabalhadores de enfermagem.

A repetitividade das ações nesse ambiente, o cansaço físico e a sobrecarga de trabalho da CME são alguns dos fatores que estimulam, nos trabalhadores, o desejo de transferência para outras unidades que permitam a convivência com o paciente e o aperfeiçoamento da habilidade psicomotora durante o cuidado direto ao cliente ${ }^{13}$. Ainda, a repetitividade das ações possibilita o aumento da prevalência das doenças relacionadas ao trabalho, como as lesões por esforços repetitivos (LER) e aos distúrbios osteomusculares relacionados ao trabalho (DORT), ampliando o sofrimento no trabalho ${ }^{3,14}$.

As LER e os DORT consistem em distúrbios funcionais ou orgânicos resultantes de fadiga de origem ocupacional. Destaca-se que essas afecções podem acometer tendões, sinovias, fáscias, ligamentos, de forma isolada ou associadamente, com ou sem degeneração de tecidos, atingindo, principalmente, os membros superiores, região escapular e pescoço. É de origem ocupacional, decorrente da forma combinada ou não do uso repetitivo e forçado de grupos musculares, bem como a manutenção de postura inadequada ${ }^{15}$.

Os participantes também descrevem que vivenciam situações geradoras de estresse ocupacional provocadas por diferentes fatores: as dificuldades impostas pela organização do trabalho, como a escassez de material e de recursos humanos; o desconhecimento por parte das outras unidades assistenciais sobre as 
atividades desenvolvidas pela CME, desvalorizando o trabalho nesse local; e o ritmo intenso de trabalho:

O nível de estresse aqui é muito alto, até pelas próprias dificuldades do serviço, porque falta enzimático, falta papel higiênico, tudo interfere na saúde. Então, esta é a razão pelo qual eu falei que tenho enxaqueca, insônia, estou hipertensa, tudo por conta deste nível de estresse, $e$ ainda é pior porque as pessoas não conhecem o trabalho da CME. (E1)

A gente confronta situações nas quais o nível de estresse eleva, a pressão eleva e a gente tenta controlar da melhor forma possível. Esse estresse é pela falta de recursos materiais e de pessoal e pela alta responsabilidade do enfermeiro neste trabalho. (E5)

O estresse ocupacional pode ocorrer em decorrência da complexidade das relações entre, de um lado, as condições laborais e extralaborais, e, de outro, as características do trabalhador. Assim, há desgaste e/ou redução da capacidade do organismo para o trabalho, oriundo da dificuldade de tolerância, de superação ou de adaptação às exigências de natureza psicológica percebidas como abusivas, insuperáveis e inesgotáveis. As repercussões da exposição ao estresse laboral manifestam-se pelo desencadeamento de outras doenças, como hipertensão, enxaqueca e úlceras gástricas, que alertam para a necessidade de modificações na organização laboral e de implementação de ações em prol da promoção da saúde desses trabalhadores ${ }^{16,17}$.

Nesse sentido, o estresse afeta a dimensão psíquica, o que acaba levando ao desgaste físico do trabalhador, provocando doenças e bloqueando a capacidade criativa e de elaboração de soluções para problemas laborais. Toda essa circunstância não só prejudica a concentração e a atenção nas atividades, mas também diminui ou elimina a satisfação em poder trabalhar e em ser produtivo e útil ${ }^{18}$.

Outra situação relevante é o calor excessivo ao qual os trabalhadores estão expostos, diariamente, durante o turno de trabalho.

Aqui é muito quente, faz mais de quarenta graus. Faz muito calor! Se fosse mais confortável em termos de temperatura, as pessoas teriam mais disposição para trabalhar. (TE3)

A exposição aos riscos físicos devido às altas temperaturas e à ventilação deficiente prevalece nos discursos, o que foi constatado no decorrer da observação de campo, já que grande parte dos subsetores estava muito quente, prejudicando não somente a saúde dos trabalhadores, mas também o armazenamento dos materiais.

O desconforto térmico pode causar danos à saúde dos trabalhadores, acarretando a diminuição da capacidade de concentração e o aumento da fadiga, além de favorecer a incidência de acidentes. É preciso salientar que as temperaturas elevadas não são adequadas ao ambiente de trabalho em saúde, pois a proliferação de micro-organismos se acelera com o calor, elevando o risco de infecções ${ }^{19}$.

Outra questão citada pelos participantes é a alta exposição ao risco biológico, relacionada, principalmente, ao manuseio de material perfurocortante contaminado.

Às vezes, o pessoal manda material perfurocortante contaminado, sem nenhum cuidado. Então, temos um grande risco de acidente. Semana passada veio um cabo de bisturi montado, e, às vezes, o paciente é portador de hepatites, HIV, e não vem sinalizado nos rótulos. A gente manipula os materiais, e podemos nos contaminar. (TE13)

O manuseio de artigos contaminados por material biológico requer grande atenção e cuidado por parte dos profissionais. Para isso, devem ser estabelecidas medidas-padrão de precauções, independentemente do nível de contaminação do artigo. Ademais, o uso de equipamentos de proteção individual (EPI) é essencial na prevenção de acidentes e na proteção do profissional contra a contaminação, conferindo ao trabalhador maior segurança no seu ambiente laboral ${ }^{20}$.

Logo, os trabalhadores que atuam nos serviços de saúde têm possibilidades de adquirir enfermidades e sofrer acidentes de trabalho em decorrência da exposição aos múltiplos fatores de risco, especialmente os de natureza biológica, física e ergonômica ${ }^{20}$.

Nessa perspectiva, o trabalho nos ambientes hospitalares tem sido arriscado e insalubre, e tem levado os trabalhadores a realizarem suas tarefas, por vezes, de maneira inadequada, sem o uso dos EPI ou sem condições laborais adequadas, decorrentes da estrutura física inapropriada. Tais fatores comprometem a rotina de trabalho e a qualidade de vida do indivíduo em seu ambiente laboral, levando-o a acidentar-se ${ }^{21}$.

É importante que se reflita sobre o processo de trabalho e a saúde do trabalhador na CME, já que é possível constatar a presença de riscos ocupacionais específicos da atividade e o quão vulneráveis são os trabalhadores de enfermagem a essas condições.

\section{Fatores que resgatam e asseguram a saúde do trabalhador}

Aqui, discutem-se situações laborais que ajudam o trabalhador a assegurar a saúde, porque elas traduzem a satisfação pessoal e o sentimento de utilidade, que ajudam o trabalhador a se precaver de doenças e a superar limitações preexistentes por conta de especificidade do trabalho desenvolvido. No entanto, há questões que, na perspectiva do trabalhador, são entendidas como boas para a saúde, mas que, se confrontadas com a literatura, evidenciam uma visão equivocada e distorcida do sujeito.

São declarações obtidas: 
A gente batalha muito para conseguir melhoria na qualidade dos nossos insumos. E, quando conseguimos isto, é gratificante, dá uma sensação de que você fez seu trabalho. (E1)

A gente, trabalhando lá em cima [subsetor esterilização], a gente faz exercício, porque pegamos caixas, andamos de um lado para o outro, então fazemos uma ginástica. (TE18)

Devido às suas características, o processo e a organização do trabalho são tão alienantes que embotam a capacidade de reflexão e julgamento do trabalhador. Desse modo, o que a literatura interpreta como prejudicial à saúde acaba sendo, distorcidamente, percebido pelo trabalhador como salutar ${ }^{22}$. O depoimento do último depoente configura-se como um claro exemplo dessa análise, pois carregar peso cotidianamente, sem orientação ergonômica nem equipamentos adequados, e ainda percorrer constantemente longas distâncias, com calçados e roupas inadequadas e sem a consideração acerca das especificidades biopsicossociais do trabalhador, é fator que propicia o desencadeamento de doenças.

Os funcionários da CME não têm um programa de assistência efetiva voltada para a saúde do trabalhador, a fim de proporcionar ginástica laboral durante algum período da jornada de trabalho. Portanto, há um equívoco no pensamento do profissional ao relacionar o levantamento de peso e as longas caminhadas durante a jornada laboral como um fator positivo para a saúde.

Nesse contexto, os programas de promoção da saúde e melhoria da qualidade de vida, os quais incorporam a ginástica laboral como atividade promotora da saúde física e mental dos trabalhadores, são indispensáveis e devem fazer parte da cultura das instituições. Tal ginástica previne problemas oriundos do exercício laboral que, em condições inadequadas, pode ocasionar grandes males à saúde dos trabalhadores ${ }^{23}$.

$\mathrm{Na}$ observação de campo, constatou-se que o coletivo profissional da CME, frequentemente, eleva peso e percorre longas distâncias, sobrecarregado com peso. Entretanto, por meio das entrevistas, verificou-se que certos sujeitos acreditam que não há necessidade de praticar exercícios físicos no ambiente extralaboral e que não é importante ter um programa privilegiando a prática de ginástica laboral na unidade, já que os profissionais acabam se movimentando durante o turno de trabalho, mesmo que seja sem quaisquer orientações por parte de um profissional especialista na área.

Outra distorção na percepção de certos sujeitos diz respeito à satisfação em conseguir material para trabalhar. No entanto, isso não deve ser considerado um fator positivo, uma vez que o trabalhador deve ser munido de recursos materiais para a prática de seu exercício laboral e não deve ter sentimento de gratificação por ter conseguido insumos para trabalhar, o que é uma obrigação da organização do trabalho e não do trabalhador, que precisa ter recursos e infraestrutura para dar conta das tarefas que lhe são prescritas pela organização laboral, conforme a RDC n ${ }^{0}$ 63/201124.

Além disso, há o relato de que, atualmente, o emprego das novas tecnologias no ambiente de trabalho estudado tem contribuído para melhorar ou assegurar a saúde dos trabalhadores do setor, configurando-se em outra visão distorcida dos sujeitos, já que, durante as observações de campo, constatou-se uma precariedade de recursos materiais e pouca inovação tecnológica.

Hoje em dia, as coisas melhoraram para a nossa saúde, porque a tecnologia ajudou muito, temos equipamentos mais fáceis de manejar, rápidos e mais leves, por exemplo. (TE8)

Na área da saúde, tem-se observado um acelerado processo de transformação e inovação tecnológica, caracterizado pela introdução de grande volume de técnicas, instrumentos, recursos diagnósticos e terapêuticos. Esse fenômeno tem produzido mudanças significativas nas práticas de saúde cotidianas e nas formas de organização e reorganização dos serviços de saúde ${ }^{25}$. Entretanto, diante da observação realizada no setor, constatou-se que os trabalhadores convivem com equipamentos de difícil manejo, arcaicos e muito pesados, distanciando-se, assim, do uso de tecnologias inovadoras que beneficiam a saúde dos trabalhadores, contrariando o último.

Fica evidente, por meio da observação, que a CME estudada ainda se encontra em processo de modernização. Apesar de possuir alguns maquinários mais novos, como as autoclaves e a lavadora ultrassônica, o setor ainda está muito aquém do que poderia oferecer aos trabalhadores, se comparado a outras CME já visitadas e se consultados os manuais de empresas que produzem tais tecnologias.

Alguns participantes indicam que o ambiente de trabalho, na forma como está constituído, favorece o cooperativismo entre os pares, o que é percebido como fator que propicia a saúde.

O ambiente de trabalho aqui é bom, a equipe está sempre disposta a ajudar, são pessoas muito boas, buscam soluções quando os problemas aparecem. (E1)

Aqui a gente nunca deixa o pessoal trabalhar sozinho, temos uma parceria incrível. Se tiver precisando de alguém lá na frente, a gente ajuda, e vice-versa. (TE18)

Essa ajuda mútua entre os trabalhadores do setor foi amplamente constatada durante o período de observação de campo. As diferentes habilidades de cada membro são empregadas de forma a alcançar o resultado almejado e, no ambiente de trabalho, destacam-se o respeito e o estímulo à criatividade ${ }^{26}$. Assim, os trabalhadores da CME possuem tal entendimento de equipe e se beneficiam positivamente da coesão entre seus integrantes. 


\section{ConClusão}

Considera-se que os trabalhadores de enfermagem atuam em condições indignas, nas quais convivem com os mais diversificados tipos de riscos ocupacionais. Assim, torna-se relevante a sensibilização dos gestores e trabalhadores para esse difícil contexto de trabalho, o qual claramente conduz às manifestações indesejáveis na saúde e até ao desenvolvimento de doenças nos trabalhadores. É fundamental que sejam implementadas melhorias para fomentar a qualidade de vida no trabalho, investindo-se, por exemplo, na climatização e na reestruturação da área física dos ambientes laborais e que seja incentivada a participação efetiva dos trabalhadores nas lutas políticas que envolvem a classe e a área da saúde, buscando condições dignas de trabalho.

Destaca-se, também, a importância da ampliação dos espaços de discussão entre os trabalhadores, os gestores e as entidades de classe, bem como o aumento de pesquisas científicas nessa área. Desse modo, incentivam-se e incrementam-se a capacidade crítica e reflexiva dos trabalhadores, ajudando-os a pensar estratégias de melhorias das condições de trabalho e fortalecendo-os na implementação de tais estratégias.

No que tange às limitações do estudo, há a impossibilidade de generalização dos resultados obtidos para outros contextos de trabalho, considerando que se trata de um grupo de trabalhadores específicos e limitado a um único hospital.

\section{REFERÊNCIAS}

1.Ascari RA, Vidori J, Moretti CA, Perin EMF, Silva OM, Buss E. O processo de esterilização de materiais em serviços de saúde: uma revisão integrativa. Braz J Surg Clin Res. 2013; 4:33-8.

2.Espindola MCG, Fontana RT. Riscos ocupacionais e mecanismos de autocuidado do trabalhador de um centro de material e esterilização. Rev Gaucha Enferm. [online]. 2012 [citado em 23 mar 2015]; 33:116-23. Disponível em: http://www.scielo.br/scielo.php?script=sci_arttext \&pid=S1983-14472012000100016.

3.Paschoalin HC. Presente no trabalho, mesmo doente: o presenteísmo na enfermagem. [tese de doutorado]. Rio de Janeiro: Universidade Federal do Rio de Janeiro; 2012. 4.Gonçalves FGA, Souza NVDO, Pires AS, Santos DM, D'Oliveira CAFB, Ribeiro LV. Modelo neoliberal e suas implicações para a saúde do trabalhador de enfermagem. Rev enferm UERJ. 2014; 22:519-25.

5.Assunção AA, Brito J. Trabalhar na saúde: experiências cotidianas e desafios para a gestão do trabalho e do emprego. Rio de Janeiro: Fiocruz; 2011.

6.Ministério da Saúde (Br). Agência de Vigilância Sanitária. Resolução - RDC N ${ }^{\circ} 15$, de 15 de março de 2012. Dispõe sobre requisitos de boas práticas para o processamento de produtos para saúde e dá outras providências. Brasília (DF): ANVISA; 2012.
7.Ouriques CM, Machado ME. Enfermagem no processo de esterilização de materiais. Texto contexto - enferm. [online]. 2013 [citado em 23 mar 2015]; 22:695-703. Disponível em: http://www.scielo.br/pdf/tce/v22n3/v22n3a16.pdf

8.Paschoalin HC, Griep RH, Lisboa MTL. A produção científica sobre o presenteísmo na enfermagem e suas repercussões no cuidado. Rev APS. 2012; 15:306-11.

9.Castro MR, Farias SNP. A produção científica sobre riscos ocupacionais a que estão expostos os trabalhadores de enfermagem. Esc Anna Nery. 2008; 12:364-9.

10.Ministério da Saúde (Br). Conselho Nacional de Saúde. Comissão Nacional de Ética em Pesquisa. Resolução $\mathrm{n}^{\circ} 466$ de 12 de dezembro de 2012: diretrizes e normas regulamentadoras de pesquisas envolvendo seres humanos. Brasília (DF): CNS; 2012.

11.Bardin L. Análise de conteúdo. $3^{\mathrm{a}}$ ed. Lisboa $(\mathrm{Pt})$ : Edições 70; 2011.

12.Lelis CM, Battaus MRB, Freitas FCT, Rocha FLR, Marziale MHP, Robazzi MLCC. Distúrbios osteomusculares relacionados ao trabalho em profissionais de enfermagem: revisão integrativa da literatura. Acta Paul Enferm. [on line]. 2012 [citado em 23 mar 2015]; 25:47782. Disponível em: http://www.scielo.br/scielo.php?scrip$\mathrm{t}=$ sci_arttext\&pid $=\mathrm{S} 0103-21002012000300025$.

13.Gallas SR, Fontana RT. Biossegurança e a enfermagem nos cuidados clínicos: contribuições para a saúde do trabalhador. Rev Bras Enferm. [on line]. 2010 [citado em 23 mar 2015]; 63:786-92. Disponível em: http://www.scielo.br/scielo.php?script=sci_arttext\&pi$\mathrm{d}=$ S0034-71672010000500015.

14.Alencar MCB, Ota NH. O afastamento do trabalho por LER/DORT: repercussões na saúde mental. Rev Ter Ocup. Univ. São Paulo. 2011; 22:60-7.

15.Pereira SMS, Brasileiro ME, França RV. Problemas de saúde que podem surgir com a repetição do trabalho. Revista Eletrônica de Enfermagem do Centro de Estudos de Enfermagem e Nutrição [serial on-line] 2011; [citado em 15 mar 2015] 1:1-15. Disponível em: http://www. ceen.com.br/revistaeletronica.

16.Lorenz VR, Benatti MCC, Sabino MO. Burnout e estresse em enfermeiros de um hospital universitário de alta complexidade. Rev Latino-Am Enfermagem [online]. 2010 [citado em 23 mar 2015]; 18:1084-91. Disponível em: http//:www.scielo.br/pdf/rlae/v18n6/pt_07.pdf.

17.Hanzelmann RS, Passos JP. Imagens e representações da enfermagem acerca do stress e sua influência na atividade laboral. Rev esc enferm USP [online]. 2010 [citado em 23 mar 2015]; 44:694-701. Disponível em: http://www.scielo.br/scielo.php?script $=$ sci_arttext\&pi$\mathrm{d}=$ S0080-62342010000300020

18.Souza NVDO, Santos DM, Ramos EL, Anunciação CT, Thiengo PCS, Fernandes MC. Repercussões psicofísicas na saúde dos enfermeiros da adaptação e improvisação de materiais hospitalares. Esc Anna Nery [on line]. 2010 [citado em 23 mar 2015]; 14:236-43. Disponível em: http://www.scielo.br/scielo.php?script=sci_arttex$\mathrm{t} \&$ pid $=$ S1414-81452010000200005.

19.Bessa MEP, Almeida MI, Araujo MFM, Silva MJ. Riscos ocupacionais do enfermeiro atuante na estratégia 
de saúde da família. Rev enferm UERJ. 2010; 18:644-9. 20.Guimarães EAA, Araujo GD, Bezerra R, Silveira RC, Oliveira VC. Percepção de técnicos de enfermagem sobre o uso de equipamentos de proteção individual em um serviço de urgência. Cienc enferm. 2011; 17:113-23. 21.Giomo DB, Freitas FCT, Alves LA, Robazzi MLCC. Acidentes de trabalho, riscos ocupacionais e absenteísmo entre trabalhadores de enfermagem hospitalar. Rev enferm UERJ. 2009; 17:24-9.

22.Dejours C, Abdoucheli E, Jayet C. Psicodinâmica do trabalho: contribuições da escola dejouriana à análise da relação prazer, sofrimento e trabalho. São Paulo: Atlas; 2011.

23.Ferreira KS, Santos AP. Os benefícios da ginástica laboral e os possíveis motivos da não implantação. Revista
Educação Física UNIFAFIBE. [on line]. 2013 [citado em 23 mar 2015]; 2.56-72. Disponível em: http//:unifafibe. com.br/revistasonline/arquivos/.../29/16122013151810.pdf. 24.Ministério da Saúde (Br). Agência Nacional de Vigilância Sanitária. Resolução da Diretoria Colegiada $n^{\circ}$ 63, de 25 de novembro de 2011. Brasília (DF): AN. VISA; 2011.

25.Caetano R, Vianna CMM. Processo de inovação tecnológica em saúde: uma análise a partir da organização industrial. Cad Saúde Coletiva. 2006; 14:95-112.

26.Navarro ASS, Guimarães RLS, Garanhani ML. Trabalho em equipe: o significado atribuído por profissionais da estratégia de saúde da família. Rev Min Enferm. [online]. 2013 [citado em 23 mar 2015]; 17:61-8. Disponível em: http://www.reme.org.br/artigo/detalhes/579. 